\title{
European Network of Clinical Research in Ophthalmology Information Update - December 2018
}

\author{
Cecília V. Martinho Daniel Sanches Fernandes
}

EVICR.net, AIBILI, Coimbra, Portugal

\section{Keywords}

Multinational clinical research

EVICR.net is a network of European ophthalmological clinical research centers dedicated to performing multinational clinical research in ophthalmology with the highest standards of quality, following the European and International regulations for clinical research and $\mathrm{ICH}-$ GCP guidelines.

EVICR.net aim is to strengthen the capacity of the European Union to perform multinational clinical research studies to develop and optimize the use of diagnostic, prevention, and treatment strategies in ophthalmology, contributing to bring better patient care to daily clinical practice. EVICR.net offers a unique platform for ophthalmology multinational clinical research in Europe and a useful industry resource in order to contribute to the development of new drugs, gene and cell therapy products, medical devices, and biomarkers.

AIBILI, in Coimbra, Portugal, is the headquarters and coordinating center of the European Vision Institute Clinical Research Network - EVICR.net. At present, EVICR.net has 105 clinical ophthalmological centers as

\section{KARGER}

(C) 2019 S. Karger AG, Basel

E-Mail karger@karger.com

www.karger.com/ore members from 15 European countries (Fig. 1). The Network has coordinated already 17 studies and has at present 6 multinational clinical studies ongoing, of which 2 are funded by the European Commission (Fig. 2).

Any clinical research center in ophthalmology can apply for EVICR.net membership. In order to become a member, basic requirements need to be fulfilled, such as adequate facilities to perform clinical research studies, qualified and experienced personnel, experience in multinational clinical studies, and willingness to implement organizational standard operating procedures (SOPs) according to the ICH-GCP guidelines. EVICR.net is the perfect partner for the coordination of multinational investigator-initiated research (IIR), particularly when applying for European Commission funding or industry IIR grants.

Scientifically, it is organized by ophthalmology subspecialties expert committees, namely: age-related macular degeneration; retinal dystrophies (including pediatric ophthalmology); diabetic retinopathy and vascular diseases; glaucoma; anterior segment, and ocular surface, inflammation, dry eye and allergies. It also has transversal sections dedicated to reading center, rare diseases, and medical devices. 
Fig. 1. Number of members per country.

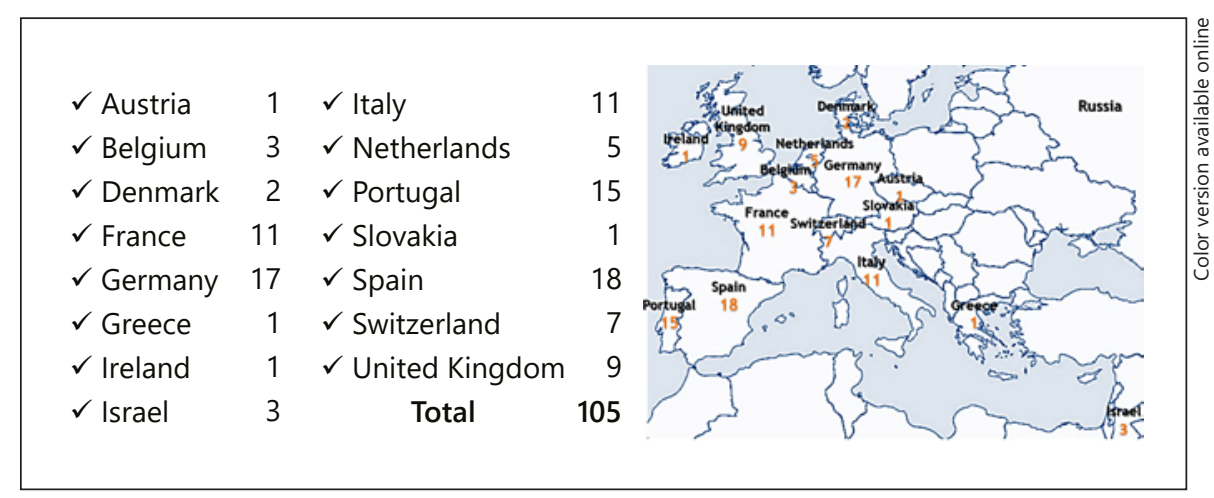

Fig. 2. Cumulative multinational clinical research studies under EVICR.net (20102018).

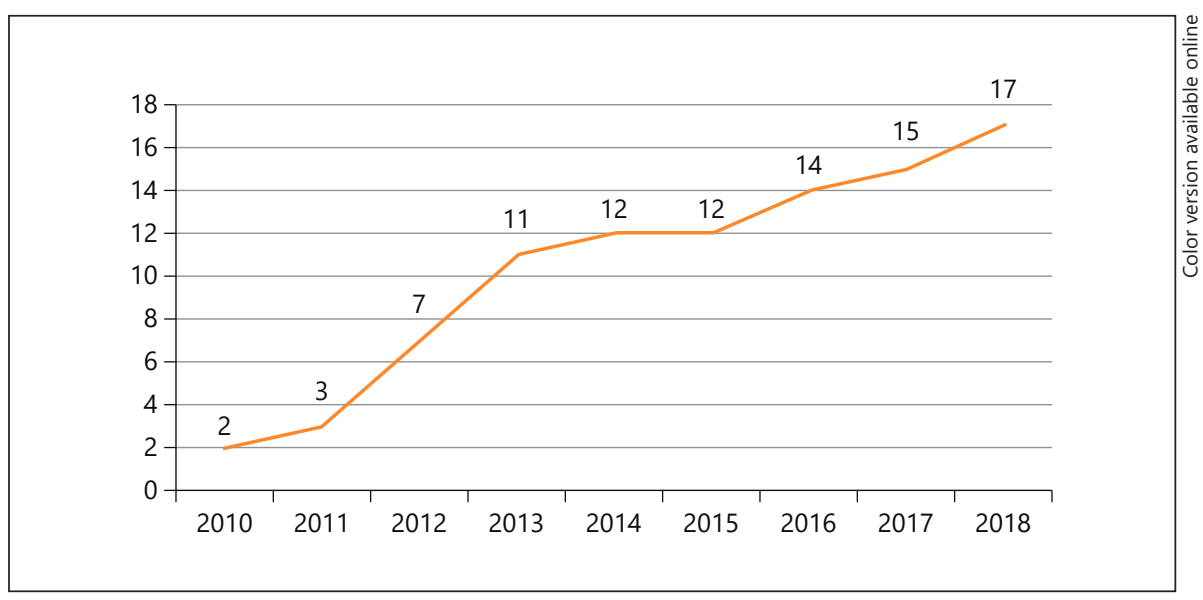

The coordinating center, AIBILI, is the single contact point for the members and industry when performing multinational ophthalmological clinical research in Europe. Annually, the coordinating center updates the EVICR.net research resources directory, where all members have their resources listed, namely: staff, equipment, and facilities as well as their scientific areas of clinical research and their five most relevant publications. All members have access to a restricted area of the website (www. evicr.net), which is also kept updated by the coordinating center. The coordinating center is responsible for the certification of EVICR.net clinical site members.

\section{Clinical Studies and Registries}

The EVICR.net coordinating center assumes the coordination and management of IIR in ophthalmology across Europe through the network. EVICR.net members have the opportunity to participate in IIR within the network as well as to submit abstracts for IIR, which are evaluated by a specific expert committee. When approved, they will have access to support for coordinating and implementing the study.

EVICR.net IIR has been growing in the last years, giving investigators opportunity to perform multinational clinical research of high quality in compliance with $\mathrm{ICH}$ GCP guidelines, assuming that the rights, safety, and wellbeing of the trial subjects are protected and that the clinical data are credible. EVICR.net has contributed to the improvement of diagnostic, prevention, and treatment strategies in ophthalmology.

\section{Educational Program}

EVICR.net launched an educational program on 2017/2018 with the aims of making educational webinars on ophthalmology clinical research available and share its experience and expertise with the clinical research community (Fig. 3). Upon completion of the webinars, participants should be able to participate more effectively in 


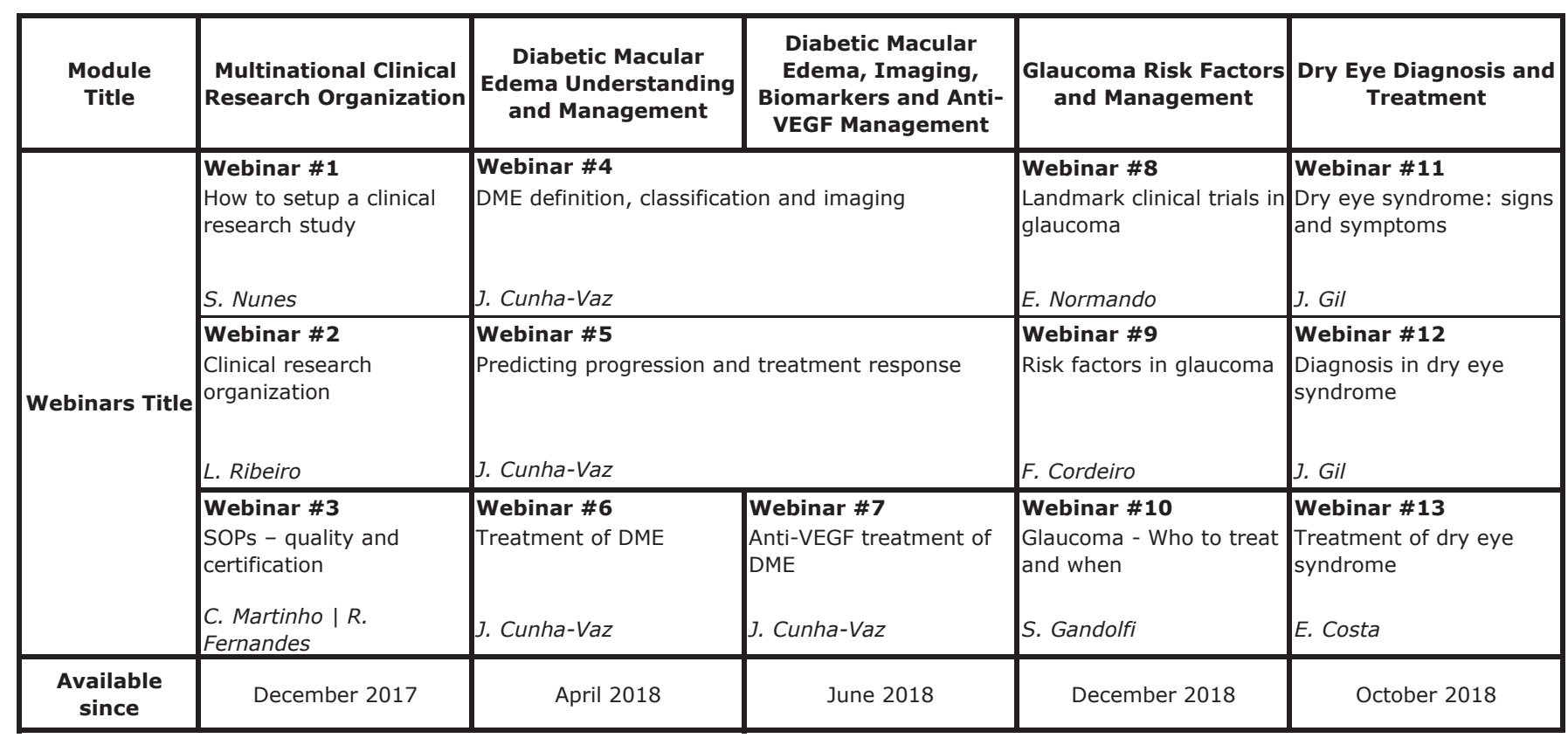

Fig. 3. Educational program webinars launched in 2017/2018.

clinical research studies in the specific scientific areas presented and become familiar with the EVICR.net certification process and how to implement and keep their organizational SOPs updated. The EVICR.net educational program is organized by modules and each module has 3 webinars. They are performed by well-known experts in their scientific area.

\section{Standard Operating Procedures - Quality System}

EVICR.net has developed a quality system for its members compliant with the ICH-GCP guidelines. It provides to its members 9 organizational SOPs for free. All our clinical site members agree to adopt these SOPs in their centers, which will be checked before they are certified as sites of excellence. The implementation of these 9 organizational SOPs will permit the clinical sites to have a standard way of working in compliance with the ICH-GCP guidelines when performing clinical research.

EVICR.net has also developed 31 technical SOPs for performing specific ophthalmic examinations or evaluations that can be used within the network for clinical studies. These SOPs are also made available to the members.

European Network of Clinical Research in Ophthalmology Information Update
In parallel, EVICR.net has developed 22 organizational SOPs for the reading centers so they can work as a network of reading centers and be able to have the capacity to respond to the industry needs for grading ophthalmological images in a standardized way with the most novel equipment.

\section{European Reference Network on Rare Eye Diseases - ERN-EYE}

The European Reference Network on Rare Eye Diseases (ERN-EYE) is led by Prof. Hélène Dollfus (Strasbourg, France) and is composed of 29 healthcare providers from 13 European countries. EVICR.net is a member of the scientific, medical and ethical advisory board. ERN-EYE is organized in thematic groups: Retinal; neuro-ophthalmology; pediatric ophthalmology, and anterior segment. EVICR.net collaborates with ERN-EYE through the rare diseases transversal section and with experience in quality systems and certification of clinical sites as well as by providing overall management and logistical support needed in multinational investigator-initiated studies in rare eye diseases. 


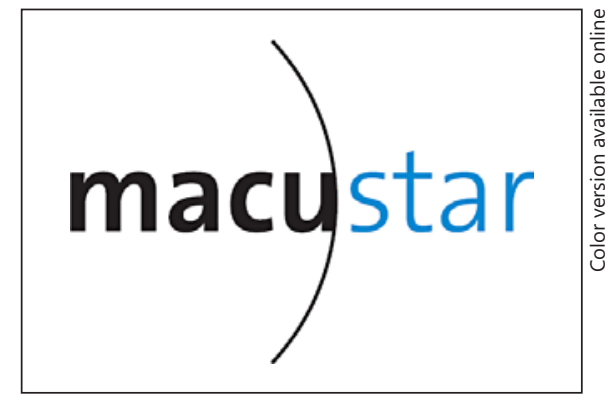

Fig. 4. MACUSTAR logo.

\section{ECRIN - European Clinical Research Infrastructure Network}

EVICR.net, as a disease-oriented network in ophthalmology for clinical research, is an affiliate partner of ECRIN-ERIC, an organization that facilitates the development and implementation of multinational clinical studies in Europe. EVICR.net serves as a resource to ECRIN-ERIC in the area of vision and ophthalmology clinical research, particularly providing scientific and medical expertise, access to patients, and clinical research capacity in ophthalmology through its members in an organized manner and compliant with the ICH-GCP guidelines and the European regulation for clinical research. An example of this partnership is the MACUSTAR study (Fig. 4), where EVICR.net is responsible for the overall coordination of the clinical study and ECRIN is responsible for the submission and monitoring activities.

\section{IMI2 Project - MACUSTAR Study}

The MACUSTAR study (development of novel clinical end points for clinical trials in intermediate AMD with a regulatory and patient access intention) started pre-recruitment on 01/09/2017. It has received funding from the European Union Innovative Medicines Initiative 2 (IMI2) Joint Undertaking. The coordinating investigator is Prof. Frank Holz (EVICR.net clinical site 15) and the sponsor is the University Hospital Bonn in Germany. EVICR.net coordinating center, AIBILI, is responsible for the overall coordination of the MACUSTAR multinational clinical study. During the IMI evaluation of MACUSTAR, EVICR.net and AIBILI were recognized as the instrumental asset for the implementation of the MACUSTAR multinational clinical study with a track record of experience in successfully conducting multicenter clinical research studies.

Age-related macular degeneration (AMD) is the commonest cause of severe visual loss and blindness in Europe and affects almost $30 \%$ of the older population. The chronic, multifactorial disease progresses slowly from early AMD to intermediate AMD (iAMD) and ultimately to late-stage AMD with severe and frequently irreversible visual loss of central vision.

The objectives of MACUSTAR are to characterize the functional deficit in iAMD and to develop and validate functional, structural, and patient-reported outcome measures for iAMD. In addition, risk factors for progression from iAMD to late AMD will be investigated.

Late-stage AMD affects more than 2.5 million patients in the European Union. To date, there is no treatment to cure or delay progression of the disease. Only treatment for the late "wet" form of the disease is available, which stabilizes the disease but does not cure it. To reduce severe visual impairment and blindness due to AMD, novel therapies are needed. MACUSTAR lays the ground for innovative drug development by developing and validating novel clinical endpoints for future clinical trials in iAMD to gain new insights into the progression from iAMD to late AMD.

MACUSTAR's core is an observational multinational clinical study with 20 EVICR.net clinical sites from $7 \mathrm{Eu}$ ropean countries, in which 750 participants will be recruited and reviewed for a period of up to 3 years. The recruitment started on 26/03/2018 and is ongoing. The clinical study, in which candidate endpoints are assessed, has two parts - a cross-sectional and a longitudinal part. The cross-sectional part assesses measurement characteristics of the candidate endpoints in early, intermediate, late, and no AMD as well as their ability to discriminate between the different groups. The second part of the study examines the progression within intermediate AMD as well as from iAMD to late-stage AMD against the functional, morphological, and patient-reported alterations. This will lay the groundwork for the development and validation of appropriate clinical endpoints for future clinical trials and drug development in iAMD.

MACUSTAR combines novel functional tests for the specific visual deficit characteristic for iAMD including dark-adapted microperimetry and standardized dark adaptation as well as state-of-the-art high-resolution retinal imaging including optical coherence tomography (OCT) and OCT angiography. A specifically developed MACUSTAR patient questionnaire assesses the impact of AMD on patients' everyday lives and allows determining pa- 
tient-relevant changes in functioning and quality of life for use in clinical trials.

MACUSTAR provides a toolbox to develop and evaluate the efficacy of novel AMD therapies. In doing so, MACUSTAR opens new paths for an accelerated drug development and lays the ground for vision-saving therapies to AMD patients in the near future. A summary of the study protocol has been published recently [1]. Further information on the MACUSTAR study can also be found on the website www.MACUSTAR.eu.

A dialogue has been established with the European Medicines Agency (EMA) and the Food and Drug Administration (FDA) in order to ascertain regulator relevance and acceptance of the developed tool box. This project has received funding from the IMI2 Joint Undertaking under grant agreement No. 116076. This Joint Un- dertaking receives support from the European Union's Horizon 2020 research and innovation program and EFPIA.

\section{Acknowledgements}

We appreciate the reviewing and contributions by Hendrik Scholl, Frank Holz, Robert Finger, Friedrich Asmus, and Jan Terheyden.

\section{References}

1 Finger RP, Schmitz-Valckenberg S, Schmid M, Rubin GS, Dunbar H, Tufail A, et al.; on behalf of the MACUSTAR consortium. MACUSTAR: Development and Clinical Validation of Functional, Structural, and Patient-Reported Endpoints in Intermediate Age-Related Macular Degeneration. Ophthalmologica. 2018 Aug 28:1-12. 